\title{
Electron capture detector based on a non-radioactive electron source: operating parameters vs. analytical performance
}

\author{
Erik Bunert, Ansgar T. Kirk, Jens Oermann, and Stefan Zimmermann \\ Institute of Electrical Engineering and Measurement Technology, Department of Sensors and \\ Measurement Technology, Leibniz Universität Hannover, 30167 Hannover, Germany
}

Correspondence: Erik Bunert (bunert@geml.uni-hannover.de)

Received: 29 September 2017 - Revised: 9 November 2017 - Accepted: 10 November 2017 - Published: 14 December 2017

\begin{abstract}
Gas chromatographs with electron capture detectors are widely used for the analysis of electron affine substances such as pesticides or chlorofluorocarbons. With detection limits in the low ppt $_{\mathrm{v}}$ range, electron capture detectors are the most sensitive detectors available for such compounds. Based on their operating principle, they require free electrons at atmospheric pressure, which are usually generated by a $\beta^{-}$decay. However, the use of radioactive materials leads to regulatory restrictions regarding purchase, operation, and disposal. Here, we present a novel electron capture detector based on a non-radioactive electron source that shows similar detection limits compared to radioactive detectors but that is not subject to these limitations and offers further advantages such as adjustable electron densities and energies. In this work we show first experimental results using 1,1,2-trichloroethane and sevoflurane, and investigate the effect of several operating parameters on the analytical performance of this new non-radioactive electron capture detector (ECD).
\end{abstract}

\section{Introduction}

Since their advent more than 55 years ago (Lovelock and Lipsky, 1960), electron capture detectors (ECDs) have become the most important gas chromatography (GC) detectors for the detection of electron affine compounds. Apart from the introduction of pulsed operation (Maggs et al., 1971), their basic setup has remained unchanged. Although many attempts have been made to replace the radioactive source used (mainly radioactive $\mathrm{Ni}-63$ foils) to generate thermalized electrons by ionizing the carrier gas (Wentworth et al., 1992), only the pulsed helium discharge has seen some degree of practical use.

However, in other fields, for example ion mobility spectrometry, radioactive electron sources have been successfully replaced by non-radioactive electron sources. In recent years, we developed a compact, hermetically sealed nonradioactive electron source (Cochems et al., 2015). It generates free electrons through thermionic emission inside a vacuum cavity at $10^{-5}$ mbar or below. These electrons are then accelerated and can partially transmit through a $300 \mathrm{~nm}$ thin silicon nitride membrane to atmospheric pressure (in this case 1013 mbar). By varying the acceleration voltage and filament heating current, the electron energy and density can be controlled. In first fundamental investigations our nonradioactive electron source was simply attached to a small reaction chamber with a Faraday plate detector (Bunert et al., 2017b, c). Using this non-radioactive electron source for ECDs avoids all the disadvantages related to radioactive ECDs, while allowing comparable limits of detection (LoDs).

In this work we investigate the influence of the gas flow configuration (axial vs. orthogonal flow setup) and the operating parameters of the electron source on the signal amplitude of the ECD which corresponds to the change in the detector current caused by the analyte reactions with the free electrons using a constant analyte concentration. Based on these results the effect of the remaining operating parameters is investigated, such as the influence of the collector voltage or gas flow rate on the analytical performance of our nonradioactive ECD. Furthermore, this non-radioactive ECD can also be operated in pulsed mode, leading to an improved lin- 


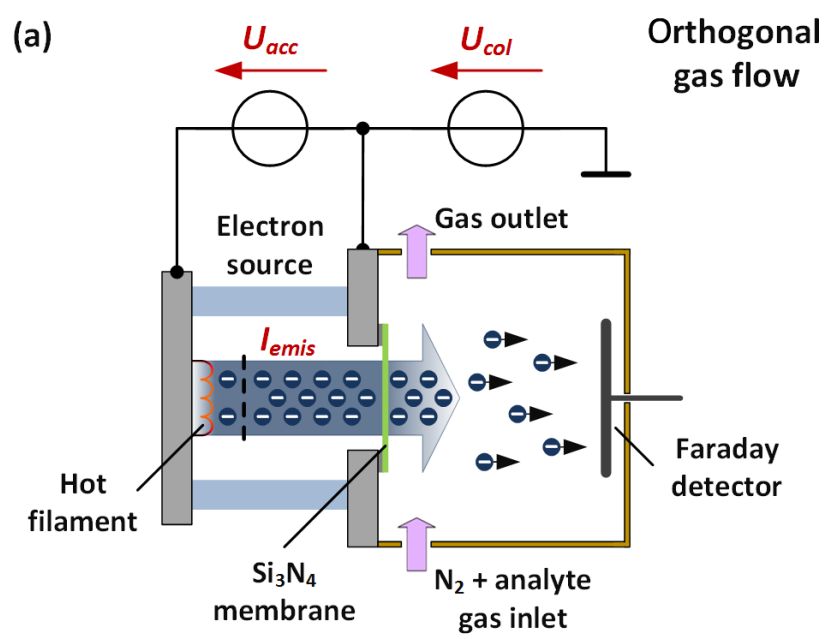

(b)

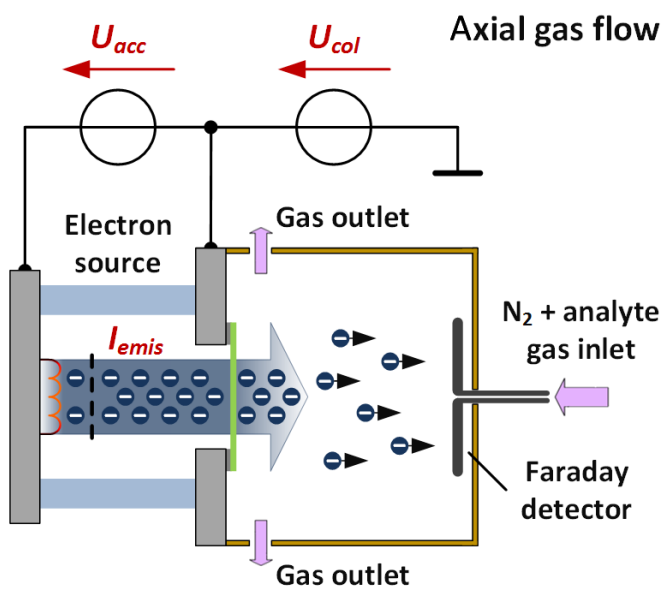

Figure 1. Schematic of our electron capture detector with our nonradioactive electron source with orthogonal (a) and axial (b) gas flow.

ear range of the sensor response compared to the typical nonlinear signal amplitude of ECDs in DC (direct current) mode (Maggs et al., 1971).

\section{Experiments}

For the first experimental investigations, our non-radioactive electron source is attached to a small reaction chamber manufactured from polyether ether ketone (PEEK). The electron source consists of a hot filament and a control grid, and is sealed with a $300 \mathrm{~nm}$ thin silicon nitride $\left(\mathrm{Si}_{3} \mathrm{~N}_{4}\right)$ membrane. The membrane is $1.5 \mathrm{~mm} \times 1.5 \mathrm{~mm}$ and is transparent for electrons with sufficient electron energy from 8 to $12 \mathrm{keV}$ (Bunert et al., 2017a). On the opposite side of the reaction chamber, a Faraday plate detector is placed. Here, two different configurations of the reaction chamber are used. In the first setup with orthogonal gas flow the sample gas (carrier gas with constant analyte concentration) passes between the electron source and the Faraday detector as shown in Fig. 1a.
In the second setup with axial gas flow the sample gas flows from the Faraday detector towards the electron source as shown in Fig. 1b. In all experiments filtered nitrogen with a dew point of $-91{ }^{\circ} \mathrm{C}$ was used as carrier gas.

To adjust the kinetic electron energy, the acceleration voltage $U_{\text {acc }}$ between the hot filament and the acceleration electrode of the electron source can be varied between 8 and $12 \mathrm{kV}$, which generates high kinetic electrons with energies between 4.5 and $9.6 \mathrm{keV}$ after transmission through the membrane (Bunert et al., 2017a). Adjusting the filament current in the range of 150 to $180 \mathrm{~mA}$ results in electron emission currents of $I_{\mathrm{emis}}=10 \mathrm{nA}$ to $I_{\mathrm{emis}}=40 \mathrm{nA}$. These primary electrons with a penetration depth of about $0.5 \mathrm{~mm}$ at $U_{\text {acc }}=8 \mathrm{kV}$ to $2.2 \mathrm{~mm}$ at $U_{\text {acc }}=12 \mathrm{kV}$ in nitrogen ionize the nitrogen molecules in the reaction region by electron impact ionization and generate secondary electrons. By applying a constant collector voltage (DC mode) of $U_{\text {col }}=3 \mathrm{~V}$ between the acceleration electrode of the electron source and the Faraday detector, the generated electrons can be collected at the Faraday detector, which constitutes a constant detector current. The collector voltage of $3 \mathrm{~V}$ is comparable to the described values in the literature (Boyes, 2010; E13 Committee, 2011) for radioactive ECDs, depending on the dimensions. The detector current is amplified by our modified transimpedance amplifier (Cochems et al., 2014) and measured by a Keysight 34461 A multimeter.

The following gas flows are given in $\mathrm{ml}_{\mathrm{S}} \mathrm{min}^{-1}$ (milliliter standard per minute, mass flow at reference conditions $20^{\circ} \mathrm{C}$ and $1013 \mathrm{mbar}$ ). The sample gas flows with a constant flow rate of $30 \mathrm{ml}_{\mathrm{s}} \mathrm{min}^{-1}$ through the reaction chamber, which is comparable to the values stated in the literature for GCs with radioactive ECDs operating in DC mode (Wagner et al., 1974). Now, the electron-capturing compounds in the carrier gas can react with the free, thermalized electrons, leading to a decreased detector current (Zlatkis and Poole, 1981).

To generate a constant analyte vapor concentration, the analyte is filled into a permeation vial heated to $35^{\circ} \mathrm{C}$ in a wellcontrolled permeation oven. This permeation oven is constantly purged with a constant flow of $600 \mathrm{ml}_{\mathrm{s}} \mathrm{min}^{-1}$ of nitrogen. An adjustable fraction of this gas is diluted with pure nitrogen to generate different analyte concentrations in nitrogen giving the sample gas. Adjustable via a mass flow controller, a fraction of this diluted sample gas can be led through the ECD as the ECD sample gas flow. For the following characterization, we used 1,1,2-trichloroethane (CAS: 79-00-5) and sevoflurane (CAS: 28523-86-6) as analytes, purchased from Sigma Aldrich.

\section{Results and discussion}

\subsection{Gas flow configuration and electron source parameters}

First, the influence of the emission current, i.e., the generated electron density, on the maximum achievable signal ampli- 
tude using a constant analyte concentration of $50 \mathrm{ppb}_{\mathrm{v}} 1,1,2$ trichloroethane was investigated. Therefore, the decrease in the detector current, which is caused by 1,1,2-trichloroethane capturing free electrons and thus corresponds to the signal amplitude, was recorded. Here, increasing the electron emission current from 10 to $40 \mathrm{nA}$ leads to an increase in signal amplitude by a factor of 3 . Unfortunately, higher emission currents cannot be investigated with our current setup, because of the limitations of the used electron source. Furthermore, a higher emission current leads to an increased offset of the detector current, which requires an increased dynamic range of the used amplifier. Thus, the highest signal amplitude for both setups (axial and orthogonal flow setup) is reached with an emission current of $40 \mathrm{nA}$.

Using this emission current of $40 \mathrm{nA}$, the influence of the electron acceleration voltage and thus the kinetic energy of the emitted electrons on the signal amplitude was investigated. An increased acceleration voltage leads to an increased penetration depth of the emitted high kinetic electrons (Bunert et al., 2017a) and hence to a larger reaction region, which should improve the achievable signal amplitude. However, with lower kinetic energies, the electrons are thermalized in less time. This is an important factor for the reaction with electron-capturing analytes and should also lead to an increased signal amplitude. The results for an acceleration voltage sweep between $U_{\text {acc }}=8 \mathrm{kV}$ and $U_{\text {acc }}=12 \mathrm{kV}$ show that the signal amplitude increases with increasing electron acceleration energies. Thus, an increasing penetration depth leading to a larger reaction region is preferable. Furthermore, electrons with higher kinetic energy can ionize more nitrogen molecules, which generates more secondary electrons. These results suggest that the maximum signal amplitude can be achieved by the highest acceleration voltage of $U_{\text {acc }}=12 \mathrm{kV}$.

However, not the signal amplitude but rather a high signalto-noise ratio (SNR) is important for lowest LoDs. Therefore, the SNR is better suited for comparing the two flow setups and was measured for different electron emission currents and electron acceleration voltages. To determine the SNR for the parameter sweeps of the electron emission current from 10 to $40 \mathrm{nA}$ and of the acceleration voltage from $U_{\mathrm{acc}}=8 \mathrm{kV}$ to $U_{\text {acc }}=12 \mathrm{kV}$, the noise of the detector signal was determined for an averaging time of $200 \mathrm{~ms}$ without analytes.

The effect of the emission current on the maximum achievable SNR for a 1,1,2-trichloroethane concentration of $50 \mathrm{ppb}_{\mathrm{v}}$ and an acceleration voltage of $U_{\mathrm{acc}}=10 \mathrm{kV}$ is shown in Fig. 2. As mentioned above, the signal amplitude increases with increasing emission current, while the noise just slightly increases. Thus, the best SNR is also reached at an emission current of $I_{\mathrm{emis}}=40 \mathrm{nA}$ for both flow setups.

However, the SNR of the axial gas flow setup is significantly higher than the SNR of the orthogonal gas flow setup for all emission currents. For example, at an emission current of $40 \mathrm{nA}$, the SNR of the axial setup is 175 and the SNR of the orthogonal setup is 130 . This can be explained by an incomplete removal of the generated analyte ions from the

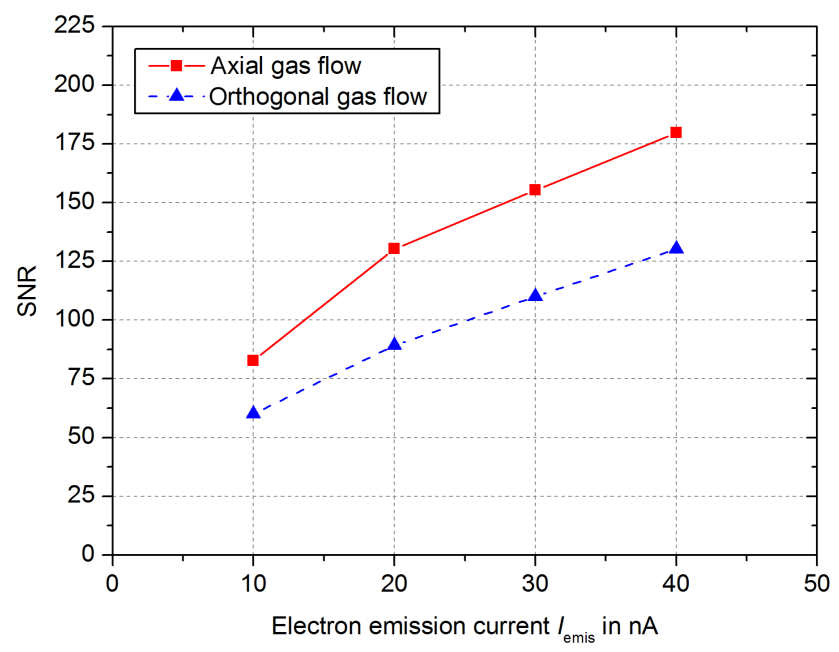

Figure 2. SNR over the electron emission current from 10 to $40 \mathrm{nA}$ with an applied collector voltage of $3 \mathrm{~V}$ and $50 \mathrm{ppb}_{\mathrm{v}} 1,1,2-$ trichloroethane. The electron source acceleration voltage is $U_{\text {acc }}=$ $10 \mathrm{kV}$.

reaction region within the orthogonal gas stream, since the electrical force on the analyte ions is directed orthogonally to the gas flow and not in the opposite direction. Thus, some charged analyte ions cannot reach the gas outlet, but can drift towards the counter electrode of the detector at the applied collector voltage. In axial configuration the convective force (gas flow velocity in the reaction region of $41 \mathrm{~cm} \mathrm{~s}^{-1}$ at $30 \mathrm{ml}_{\mathrm{s}} \mathrm{min}^{-1}$ ) exceeds the electrical force (drift velocity of $9 \mathrm{~cm} \mathrm{~s}^{-1}$ at $E=4 \mathrm{~V} \mathrm{~cm}^{-1}$ ), and analyte ions can leave the reaction region. This increases the statistical noise of the detector current, while the signal amplitude almost remains constant. However, the axial gas flow transports the analyte ions in the opposite direction of the electrical field with a slightly higher force than the electrical force by the applied collector voltage. Thus, only the electrons and not the analyte ions are detected by the Faraday detector.

Based on this, an increased acceleration voltage and thus an increased penetration depth of the emitted electrons as well as an increased number of generated secondary electrons should increase the noise by the statistical behavior of the ionization process. Therefore, the SNR for acceleration voltages between $U_{\text {acc }}=8 \mathrm{kV}$ and $U_{\text {acc }}=12 \mathrm{kV}$ was determined for both flow setups. Figure 3 shows the resulting SNR curves at a constant emission current of $I_{\mathrm{emis}}=40 \mathrm{nA}$.

The optimum SNR is 175 at $U_{\text {acc }}=10 \mathrm{kV}$ for the axial setup and, respectively, 140 at $U_{\text {acc }}=11 \mathrm{kV}$ for the orthogonal setup. Thus, the gain in signal amplitude is less than the gain in noise for acceleration voltages above $10 \mathrm{kV}$, which supports the assumption of an increased noise at higher kinetic electron energies. In summary, setting the acceleration voltage to its optimum value of $U_{\mathrm{acc}}=10 \mathrm{kV}$ and the emission current to $I_{\mathrm{emis}}=40 \mathrm{nA}$ leads to the maximum SNR of 175. Furthermore, the axial gas flow setup is preferable be- 


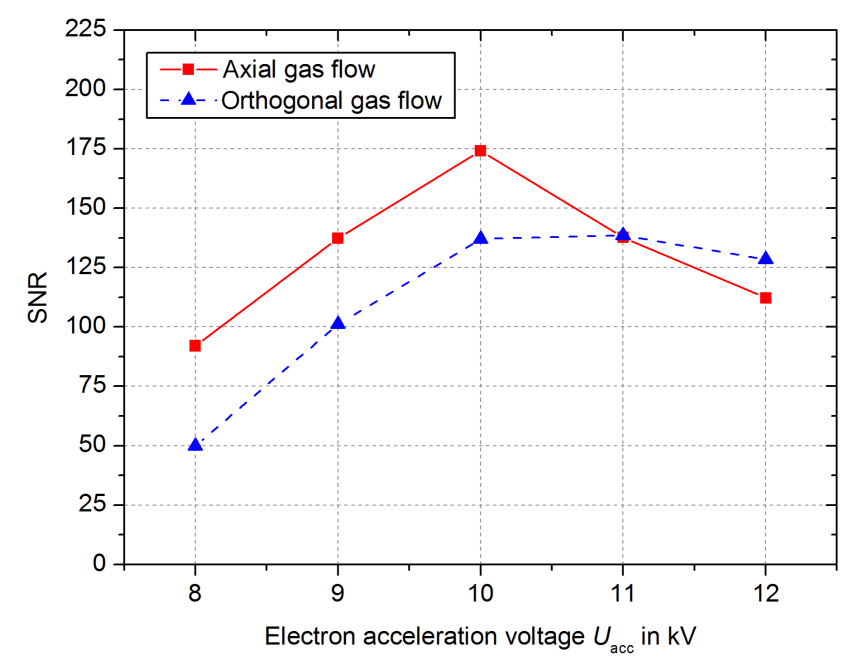

Figure 3. SNR over the acceleration voltage from 8 to $12 \mathrm{kV}$ with an applied collector voltage of $3 \mathrm{~V}$ and $50 \mathrm{ppb}_{\mathrm{v}} 1,1,2-$ trichloroethane. The electron emission current is constantly controlled to $I_{\text {emis }}=40 \mathrm{nA}$.

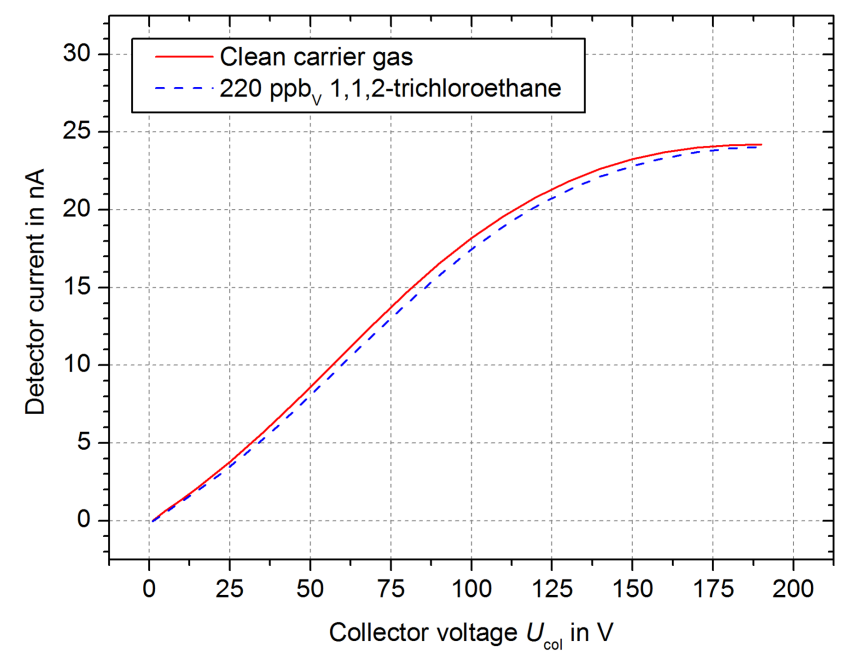

Figure 4. Detector signals with pure nitrogen as carrier gas (solid red line) and nitrogen containing $220 \mathrm{ppb}_{\mathrm{v}}$ 1,1,2-trichloroethane (dashed blue line) over collector voltage.

cause of the higher SNR and the more effective removal of analyte ions from the reaction region.

\subsection{Influence of the collector voltage}

Using the optimized electrical parameters mentioned above and the axial gas flow setup, we now investigate the effect of the collector voltage $U_{\mathrm{col}}$ on the signal amplitude. Therefore, the absolute detector current was measured for two repetitions of a collector voltage sweep between $U_{\mathrm{col}}=1 \mathrm{~V}$ and $U_{\text {col }}=190 \mathrm{~V}$, the first with pure carrier gas and the second with sample gas containing a constant 1,1,2-trichloroethane

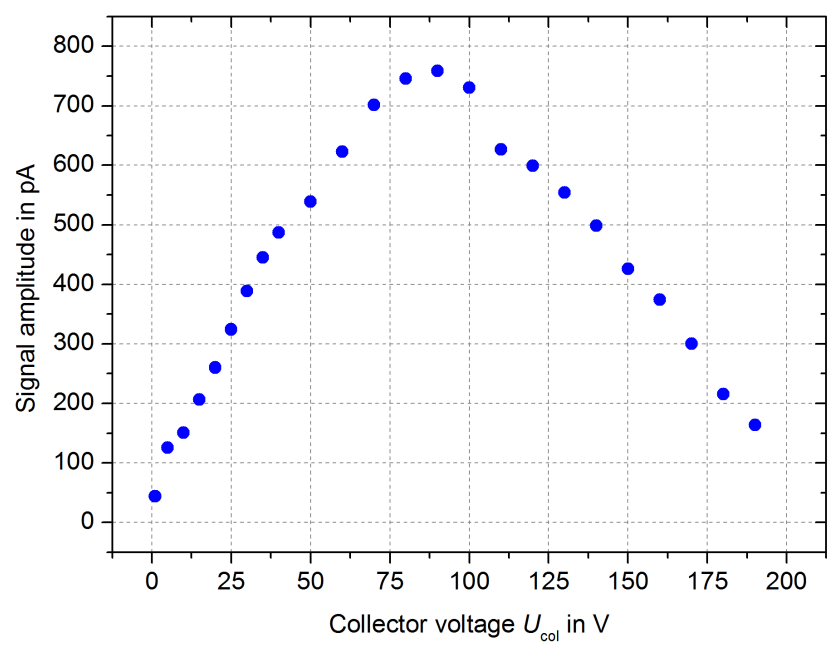

Figure 5. Signal amplitude of the detector response over collector voltage calculated by the difference between the detector signals with pure nitrogen as carrier gas and nitrogen containing $220 \mathrm{ppb}_{\mathrm{v}}$ 1,1,2-trichloroethane.

concentration of $220 \mathrm{ppb}_{\mathrm{v}}$. This relatively high concentration is mainly used to generate higher detector signals to better analyze the effect of the collector voltage on the detector signal. As explained above, the analytes capture free electrons, which causes a decrease in the detector current as shown in Fig. 4. Thus, the difference between the two curves related to the analyte concentration corresponds to the signal amplitude of this setup; see Fig. 5. The absolute detector currents for both curves saturate at the same value of $I_{\text {det,sat }}=24 \mathrm{nA}$ for higher collector voltages, independent of the analyte concentration, as all charged particles are captured at this point. In addition, the signal amplitude has a maximum value of $760 \mathrm{pA}$ at a collector voltage of $U_{\mathrm{col}}=90 \mathrm{~V}$.

Again, not the signal amplitude but rather a high SNR is important for lowest LoDs. Therefore, the SNR is calculated by dividing the signal amplitude by the noise of the detector signal for an averaging time of $200 \mathrm{~ms}$. The results in Fig. 6 show an increasing noise with an increasing collector voltage. Since the signal amplitude also increases, the SNR remains above a value of 200 up to $U_{\mathrm{col}}=100 \mathrm{~V}$. For higher collector voltages, however, the SNR significantly decreases. Furthermore, the SNR has two maxima of 280 at $U_{\mathrm{col}}=5 \mathrm{~V}$ and $U_{\text {col }}=70 \mathrm{~V}$. In the following measurements a collector voltage of $U_{\mathrm{col}}=5 \mathrm{~V}$ is used due to the reduced instrumental effort of a low voltage power supply.

Next, the influence of the sample gas flow on the resulting signal amplitude is investigated. Therefore, we used different 1,1,2-trichloroethane and sevoflurane concentrations for sample gas flows of 5,10 , and $30 \mathrm{ml}_{\mathrm{s}} \mathrm{min}^{-1}$. The results in Fig. 7 show a slightly decreased signal amplitude for lower sample gas flows. A possible explanation is again the incomplete removal of analyte ions from the reaction region within the gas stream, so that both electrons and charged analyte 


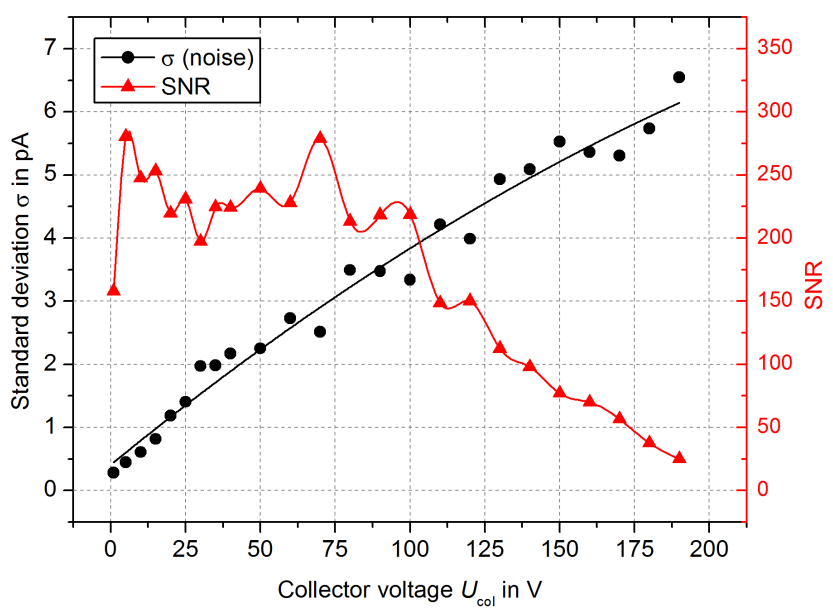

Figure 6. Standard deviation $\sigma$ of the measured noise (black dots) and the resulting signal-to-noise ratio (red triangles) over collector voltage.

molecules are detected, which reduces the signal amplitude. Furthermore, the typical non-linear response of electron capture detectors in DC mode (Maggs et al., 1971; Wagner et al., 1974) with increasing concentrations is visible.

Based on the measured linear signal response at low concentrations, we determined the limits of detection for 1,1,2trichloroethane and for sevoflurane at different sample gas flow rates. Here, the LoD is defined as 3 times the standard deviation $\sigma$ of the measured noise. The calculated LoDs are listed in Table 1 for an averaging time of $200 \mathrm{~ms}$. At a sample gas flow rate of $30 \mathrm{ml}_{\mathrm{S}} \mathrm{min}^{-1}$ the LoDs of our nonradioactive ECD are $0.005 \mu \mathrm{gL}^{-1}$ for 1,1,2-trichloroethane and $0.002 \mu \mathrm{g} \mathrm{L}^{-1}$ for sevoflurane, which is comparable to radioactive ECDs, e.g., $0.006 \mu \mathrm{g} \mathrm{L}^{-1}$ for 1,1,2-trichloroethane (Barani et al., 2006).

In addition, we investigated the effect of the sample gas flow on the LoD. Typical flow rates used in gas chromatography range between 5 and $30 \mathrm{ml}_{\mathrm{S}} \mathrm{min}^{-1}$ (Wagner et al., 1974; Zitko et al., 1971; Kapila and Aue, 1975; Nasreddine et al., 2016). At a sample gas flow rate of $5 \mathrm{ml}_{\mathrm{s}} \mathrm{min}^{-1}$, the LoDs are $0.007 \mu \mathrm{g} \mathrm{L}^{-1}$ for 1,1,2-trichloroethane and $0.004 \mu \mathrm{g} \mathrm{L}^{-1}$ for sevoflurane. These detection limits are slightly higher because of the incomplete removal of analyte ions as mentioned above.

\subsection{Implementation of the pulsed mode}

The typical non-linear response of electron capture detectors in DC mode is clearly visible with increasing concentrations (Maggs et al., 1971; Zlatkis and Poole, 1981); see Fig. 7. Thus, we implemented the well-known pulsed collector voltage mode in order to improve the linear range of the detector response (Maggs et al., 1971; E13 Committee, 2011; Gobby et al., 1980; Zlatkis and Poole, 1981). In pulsed mode, the electrons are mainly generated under field-free conditions in

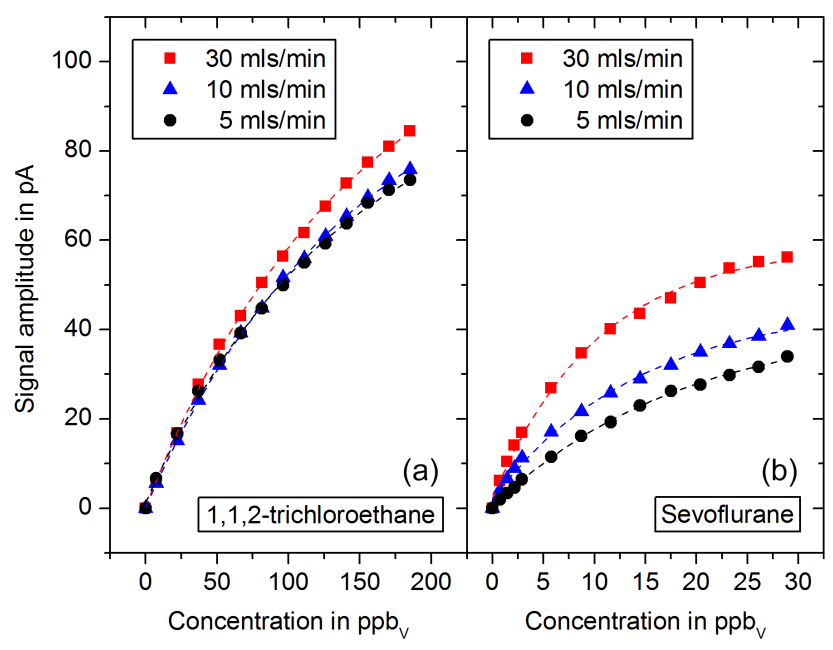

Figure 7. Resulting signal amplitudes over the analyte concentration for 1,1,2-trichloroethane (a) and sevoflurane (b) for sample gas flows of 5,10 and $30 \mathrm{ml}_{\mathrm{s}} \mathrm{min}^{-1}$.

the reaction region, while the analyte molecules react with the electrons and the analyte ions are removed within the gas stream. A short voltage pulse generates a transient electrical field in the reaction region and the remaining electrons are pushed to the Faraday detector. The voltage pulse has to be so short (typically a few $\mu$ s or less) that the charged analyte molecules with their lower mobility do not reach the detector (E13 Committee, 2011). The amplitude of this voltage pulse is typically significantly higher than in DC mode, but is not exactly described in the literature. Therefore, we set the amplitude to $80 \mathrm{~V}$. Now, the repetition rate is controlled to maintain a constant detector current, independent of the analyte concentration. The required repetition rate is a measure of the analyte concentration. Initial measurements were performed with a pulse width of $5 \mu \mathrm{s}$, a constant detector current of $7.7 \mathrm{nA}$, and a constant emission current of $I_{\mathrm{emis}}=40 \mathrm{nA}$. The results for various 1,1,2-trichloroethane concentrations are shown in Fig. 8. The linear range in pulsed mode with this preliminary setup and parameter set is between the LoD of 0.85 and $80 \mathrm{ppb}_{\mathrm{v}}$. This linear range of $10^{2}$ is just an improvement by a factor of 2 compared to the linear range of the DC mode. Further improvements, such as optimized control electronics for shorter pulse widths, are expected to significantly increase the achievable linear range up to $10^{4}$, which is typically reached by commercially available ECDs with a radioactive electron source.

\section{Conclusion}

In this work, we presented the construction and preliminary investigations of a novel electron capture detector with a nonradioactive electron source. Two different ECD configurations (axial and orthogonal flow setup) were tested. Using 1,1,2-trichloroethane as a test analyte, we found that the ax- 
Table 1. LoDs for 1,1,2-trichloroethane and sevoflurane for sample gas flows between 5 and $30 \mathrm{ml}_{\mathrm{s}} \mathrm{min}^{-1}$ for a measuring time of $200 \mathrm{~ms}$ and $U_{\mathrm{col}}=5 \mathrm{~V}$.

\begin{tabular}{lll}
\hline Sample gas flow & LoD for 1,1,2-trichloroethane & LoD for sevoflurane \\
\hline $5 \mathrm{ml}_{\mathrm{S}} \mathrm{min}^{-1}$ & $1.20 \mathrm{ppb}_{\mathrm{v}}\left(0.007 \mu \mathrm{gL}^{-1}\right)$ & $450 \mathrm{ppt}_{\mathrm{v}}\left(0.004 \mu \mathrm{gL}^{-1}\right)$ \\
$10 \mathrm{ml}_{\mathrm{S}} \mathrm{min}^{-1}$ & $1.00 \mathrm{ppb}_{\mathrm{v}}\left(0.006 \mu \mathrm{gL}^{-1}\right)$ & $340 \mathrm{ppt}_{\mathrm{v}}\left(0.003 \mu \mathrm{gL}^{-1}\right)$ \\
$30 \mathrm{ml}_{\mathrm{S}} \min ^{-1}$ & $0.85 \mathrm{ppb}_{\mathrm{v}}\left(0.005 \mu \mathrm{gL}^{-1}\right)$ & $190 \mathrm{ppt}_{\mathrm{v}}\left(0.002 \mu \mathrm{gL}^{-1}\right)$ \\
\hline
\end{tabular}

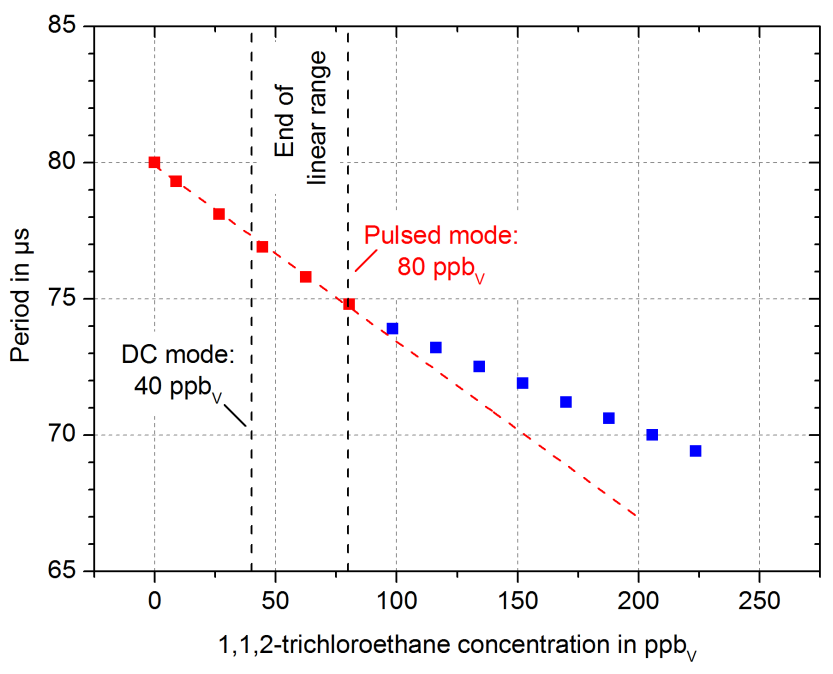

Figure 8. Extended linear range (red) for 1,1,2-trichloroethane by using a pulsed collector voltage of $U_{\mathrm{col}}=80 \mathrm{~V}$ and a pulse width of $t_{\mathrm{W}, \mathrm{col}}=5 \mu \mathrm{s}$. The pulse frequency is adjusted to keep the detector current constant independent of the analyte concentration, so that the pulse frequency and period correspond to the analyte concentration.

ial setup is superior due to the higher signal-to-noise ratio (SNR). Furthermore, we investigated the effect of different operating parameters on the SNR and thus on the limits of detection (LoDs). The SNR increases with increasing electron emission current up to the highest possible emission current of $I_{\mathrm{emis}}=40 \mathrm{nA}$. This is mainly due to the increased number of generated secondary electrons. In addition, the optimum acceleration voltage was determined to be $U_{\text {acc }}=10 \mathrm{kV}$ for the highest possible SNR. Even higher acceleration voltages lead to an increased noise because of the statistical behavior of the ionization process.

Using the parameters mentioned above together with the axial setup, further measurements were performed to optimize the collector voltage and to investigate the effect of the sample gas flow on the signal amplitude. While the noise increases with increasing collector voltage, the signal amplitude has a maximum value, leading to two SNR maxima. Due to the lower instrumental effort of a low voltage power supply, the lower collector voltage of $U_{\text {col }}=5 \mathrm{~V}$ is used to reach the maximum SNR. In order to investigate the effect of the sample gas flow on the signal amplitude, the
LoDs of 1,1,2-trichloroethane and sevoflurane were determined for different sample gas flows. At a sample gas flow rate of $30 \mathrm{ml}_{\mathrm{s}} \mathrm{min}^{-1}$ the LoDs of our non-radioactive ECD are $0.005 \mu \mathrm{g} \mathrm{L}^{-1}$ for 1,1,2-trichloroethane and $0.002 \mu \mathrm{gL}^{-1}$ for sevoflurane, which is comparable to radioactive ECDs. At a sample gas flow rate of just $5 \mathrm{ml}_{\mathrm{s}} \mathrm{min}^{-1}$, the LoDs are $0.007 \mu \mathrm{g} \mathrm{L}^{-1}$ for 1,1,2-trichloroethane and $0.004 \mu \mathrm{g} \mathrm{L}^{-1}$ for sevoflurane. Thus, the non-radioactive ECD fits the flow rates typically used in standard gas chromatography. Because of the non-linear response at high analyte concentrations, a pulsed collector voltage mode known from the literature was implemented, leading to a linear range of $10^{2}$.

Although our non-radioactive ECD already compares well with radioactive ECDs, future investigations and developments are required to further improve the analytical performance. In particular, new control electronics and shorter pulse widths should significantly expand the linear range up to $10^{4}$ as required for GC applications.

Data availability. No data sets were used in this article.

Competing interests. The authors declare that they have no conflict of interest.

Special issue statement. This article is part of the special issue "Sensor/IRS2 2017". It is a result of the AMA Conferences, Nuremberg, Germany, 30 May-1 June 2017.

Edited by: Ulrich Schmid

Reviewed by: two anonymous referees

\section{References}

Barani, F., Dell'Amico, N., Griffone, L., Santoro, M., and Tarabella, C.: Determination of Volatile Organic Compounds by Headspace Trap, J. Chromatogr. Sci., 44, 625-630, https://doi.org/10.1093/chromsci/44.10.625, 2006.

Boyes, W.: Instrumentation reference book, ButterworthHeinemann/Elsevier, Boston, 1 online resource, 2010.

Bunert, E., Heptner, A., Reinecke, T., Kirk, A. T., and Zimmermann, S.: Shutterless ion mobility spectrometer with fast pulsed electron source, Rev. Sci. Instrum., 88, 24102, https://doi.org/10.1063/1.4976021, 2017a. 
Bunert, E., Kirk, A. T., Oermann, J., and Zimmermann, S.: An Electron Capture Detector Based on a Non-Radioactive Electron Source, P3.3 - AMA Service GmbH, Von-Münchhausen-Str. 49, 31515 Wunstorf, Germany, in: Proceedings of the AMA Conferences 2017 with SENSOR and IRS ${ }^{2}$ : SENSOR 2017, 18th International Conference on Sensors and Measurement Technology, edited by: AMA Service GmbH, SENSOR 2017, Nürnberg, 30 May-1 June 2017, AMA Service GmbH, Wunstorf, 627-631, 2017b.

Bunert, E., Kirk, A. T., Oermann, J., and Zimmermann, S.: Electron Capture Detector with Non-Radioactive Electron Source, in: Proceedings, Eurosensors 2017, Paris, 3-6 September 2017, mdpi, 443, 2017c.

Cochems, P., Kirk, A. T., and Zimmermann, S.: In-circuitmeasurement of parasitic elements in high gain high bandwidth low noise transimpedance amplifiers, Rev. Sci. Instrum., 85, 124703, https://doi.org/10.1063/1.4902854, 2014.

Cochems, P., Kirk, A. T., Bunert, E., Runge, M., Goncalves, P., and Zimmermann, S.: Fast pulsed operation of a small non-radioactive electron source with continuous emission current control, Rev. Sci. Instrum., 86, 65102, https://doi.org/10.1063/1.4921707, 2015.

E13 Committee: Practice for Use of Electron-Capture Detectors in Gas Chromatography, ASTM International, West Conshohocken, PA, https://doi.org/10.1520/E0697-96R11, 2011.

Gobby, P. L., Grimsrud, E. P., and Warden, S. W.: Improved model of the pulsed electron capture detector, Anal. Chem., 52, 473482, https://doi.org/10.1021/ac50053a023, 1980.
Kapila, S. and Aue, W. A.: Voltage effects in a d.c. electron capture detector, J. Chrom. A, 108, 13-21, https://doi.org/10.1016/S0021-9673(00)97484-3, 1975.

Lovelock, J. E. and Lipsky, S. R.: Electron Affinity Spectroscopy - A New Method for the Identification of Functional Groups in Chemical Compounds Separated by Gas Chromatography 1, J. Am. Chem. Soc., 82, 431-433, https://doi.org/10.1021/ja01487a045, 1960.

Maggs, R. J., Joynes, P. L., Davies, A. J., and Lovelock, J. E.: Electron capture detector. New mode of operation, Anal. Chem., 43, 1966-1971, https://doi.org/10.1021/ac60308a014, 1971.

Nasreddine, R., Person, V., Serra, C. A., and Le Calvé, S.: Development of a novel portable miniaturized GC for near real-time low level detection of BTEX, Sens. Actuators, B, 224, 159-169, https://doi.org/10.1016/j.snb.2015.09.077, 2016.

Wagner, P. D., Naumann, P. F., and Laravuso, R. B.: Simultaneous measurement of eight foreign gases in blood by gas chromatography, J. Appl. Physiol., 36, 600-605, 1974.

Wentworth, W. E., D'Sa, E. D., Cai, H., and Stearns, S.: Environmental Applications of the Pulsed-Discharge ElectronCapture Detector, J. Chromatogr. Sci., 30, 478-485, https://doi.org/10.1093/chromsci/30.12.478, 1992.

Zitko, V., Hutzinger, O., and Safe, S.: Retention times and electroncapture detector responses of some individual chlorobiphenyls, B. Environ. Contam. Tox., 6, 160-163, 1971.

Zlatkis, A. and Poole, C. F.: Electron capture: Theory and practice in chromatography, Journal of chromatography library, v. 20, Elsevier Scientific Pub. Co, Amsterdam, New York, xii, 429, 1981. 